\title{
Influencia de Factores de Entorno en la Implementación del estándar ISO/IEC 29110
}

\author{
Stuardo Lucho ${ }^{1}$, Karin Melendez ${ }^{2}$, Abraham Dávila ${ }^{3}$ \\ stuardo.lucho@pucp.edu.pe, kmelendez@pucp.edu.pe, abraham.davila@pucp.edu.pe \\ ${ }^{1}$ Departamento de Ingeniería, Pontificia Universidad Católica del Perú, Lima 32, Lima, Perú. \\ ${ }^{2}$ Departamento de Ingeniería, Pontificia Universidad Católica del Perú, Lima 32, Lima, Perú. \\ ${ }^{3}$ Departamento de Ingeniería, Pontificia Universidad Católica del Perú, Lima 32, Lima, Perú
}

DOI: 10.17013/risti.26.1-16

\begin{abstract}
Resumen: El estándar ISO/IEC 29110 es usado por algunas empresas peruanas con el fin de mejorar la calidad del software que elaboran y ser más productivos. Sin embargo, existen factores que influyen en el equipo durante la mejora de procesos de software, por lo cual, identificar adecuadamente estos factores, permitirá mejorar el nivel de adopción en futuras implementaciones. El objetivo de este estudio es analizar los factores del entorno que influyen en la adopción del estándar ISO/IEC 29110, en su perfil básico, en 4 pequeñas empresas desarrolladoras de software, utilizando la metodología de estudio de caso múltiple. Cada empresa trabajó bajo su propio contexto y definió sus propios objetivos de mejora. Del conjunto de factores del entorno analizados, se encontró como resultado que 5 de ellos influenciaron positivamente en todas las empresas sin importar el contexto, 2 ellos negativamente y 2 de ellos tuvieron relación entre factores debido al contexto.
\end{abstract}

Palabras-clave: ISO/IEC 29110, factores de adopción, mejora de proceso software.

\section{Influence of Environment Factors in the Implementation of the ISO / IEC 29110 standard}

\begin{abstract}
The ISO/IEC 29110 standard is used by some Peruvian companies in order to improve the quality of the software they produce and be more productive. However, there are factors that influence the team during the software process improvement, for which, properly identifying these factors will allow improving the level of adoption in future implementations. The purpose of this study is to analyze the environmental factors that influence the adoption of the ISO/IEC 29110 standard, in its basic profile, in 4 small software development companies, using the multiple case study methodology. Each company worked in its own context and defined its own improvement objectives. From the set of environmental factors analyzed, it was found that 5 of them positively influenced all the companies regardless of the context, 2 of them negatively and 2 of them were related between factors due to the context.
\end{abstract}

Keywords: 29110, adoption, factors, spi. 


\section{Introducción}

Las empresas en la industria del software realizan la definición y documentación de sus actividades para: (i) gestionar sus proyectos de software de inicio a fin (Humphrey, 1995), y (ii) para proporcionar una comprensión general a todos aquellos involucrados en el desarrollo de software sobre las tareas de las cuales son responsables (Unterkalmsteiner et al., 2012). Estas actividades pueden mejorarse siguiendo diversos referentes internacionales como estándares o modelos de procesos (Vasconcellos et al., 2016) (Miramontes, Muñoz, Calvo-Manzano, \& Corona, 2016). Algunos modelos requieren mayor cantidad de recursos humanos y económicos para poder implementarlos, como el modelo CMMI o el estándar ISO/IEC 15504 (Huang \& Zhang, 2010); mientras que otros, como MoProSoft o el estándar ISO/IEC 29110, están más orientados a las pequeñas empresas artículos (O’Connor \& Laporte, 2011)(Cano, Melgar, Dávila, \& Pessoa, 2015).

$\mathrm{Al}$ adoptar un modelo de procesos, existen factores que se deben considerar para obtener beneficios o evitar retrasos, tales como el tamaño de la empresa (Horvat, Rozman, \& Györkös, 2000) (Basri \& O'Connor, 2010b), la cultura organizacional, los recursos a invertir, los resultados esperados (Ebert, 1999)(Larrucea, O'Connor, Colomo-Palacios, \& Laporte, 2010), el uso correcto de un modelo de referencia, la participación de la alta gerencia de la organización; y especialmente la alta proporción de personas que forman parte del equipo de trabajo de la organización (Basri \& O'Connor, 2010a). Todos estos factores han sido estudiados y organizados por diversos autores en categorías tales como: factores organizacionales, alta dirección y del entorno, entre otros; mientras que otros autores los han agrupado en modelos conceptuales, como los presentados en (Hameed, Counsell, \& Swift, 2012), (Bayona, Calvo-Manzano, \& Feliu, 2013), (Bayona, CalvoManzano, Cuevas, \& Feliu, 2010) y (Sulayman, Urquhart, Mendes, \& Seidel, 2012).

Con respecto a lo indicado, en el proyecto ProCal-ProSer (Díaz, De Jesús, Melendez, \& Dávila, 2016) se estudia los factores que influyen en la adopción del estándar ISO/ IEC 29110 por parte de pequeñas empresas. Los factores identificados como parte del proyecto ProCal-ProSer permitirán definir estrategias de adopción en la industria del software.

En este artículo se presenta una análisis complemetario al presentado en un artículo previo (Lucho, Melendez, \& Dávila, 2018) sobre los factores del entorno que influyen en un grupo de empresas que implementaron el estándar ISO/IEC 29110. El documento está organizado de la siguiente manera: Sección 2, muestra el marco de referencia; Sección 3, metodología de investigación; Sección 4, unidades de análisis; Sección 5, el análisis e interpretación de los resultados y la Sección 6, la discusión final y el trabajo futuro.

\section{Marco de referencia}

En este capítulo se revisaron conceptos y estudios relacionados con modelos de procesos y factores de mejora. Además, se realizó una búsqueda de trabajos similares que permitan guiarnos y realizar una investigación más completa.

\subsection{Modelos de proceso de software}

Existen diversas maneras de mejorar la calidad del producto de software, tal como se indica en los estudios (Nirmala \& LathaMaheswari, 2016), (Svensson et al., 2012) 
y (Razón, Durán, González, Cortérights, \& Miranda, 2014). Mejorar la calidad en el proceso de desarrollo de software (Elhag, Elshaikh, Mohamed, \& Babar, 2013) basado en modelos de procesos de software, es una forma de lograr mejorar la calidad en el producto.

Entre los modelos más representativos se encuentran: CMMI (SEI, 2010) y el estándar ISO/IEC 12207 (ISO/IEC, 2008). Sin embargo, ambos modelos tienen problemas de adopción y generan rechazo cuando se intenta implementar en pequeñas organizaciones (Huang \& Zhang, 2010), (Brodman \& Johnson, 1997), (Ruiz et al., 2011). Dada la situación descrita, se desarrollaron nuevos modelos que se ajustan mejor a las pequeñas empresas y algunos de ellos fueron utilizados en Perú: MoProSoft (Oktaba et al., 2005), Competisoft (Piattini, 2008), MPS.BR (Kalinowski et al., 2015); y, el estándar ISO/IEC 29110 (ISO/IEC, 2011a).

Delos modelos mencionados, la ISO/IEC 29110 es un estándar desarrollado con el objetivo de mejorar la calidad de los productos de software a través de la mejora de los procesos de las pequeñas empresas (Laporte, O’Connor, \& Paucar, 2016). El estándar define una pequeña organización (PO) como una organización, empresa, departamento o proyecto que tiene hasta 25 profesionales dedicados a proyectos de software (ISO/IEC, 2011a). El estándar está organizado por perfiles tales como: básico, intermedio y avanzado; y en cada perfil se establece un conjunto de procesos (ISO/IEC, 2011a). El perfil básico se detalla en el documento (ISO/IEC, 2011c) y está compuesto por dos procesos: (i) gestión de proyecto (GP), el cual tiene como objetivo llevar a cabo sistemáticamente las tareas del desarrollo del software, con el propósito de cumplir los objetivos del proyecto en relación con calidad, tiempo y presupuesto. (ii) Implementación del software (IS), el cual tiene como objetivo establecer la relación sistemática entre las actividades de análisis, construcción, diseño, integración y prueba del producto software según los requisitos de software.

Cada proceso y sus principales actividades se muestran en la Figura 1 y la Figura 2.

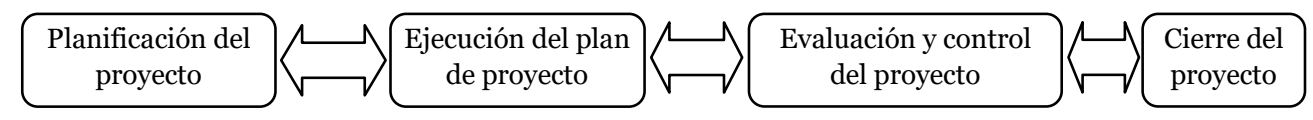

Figura 1 - Actividades principales del proceso de GP (ISO/IEC, 2011c)

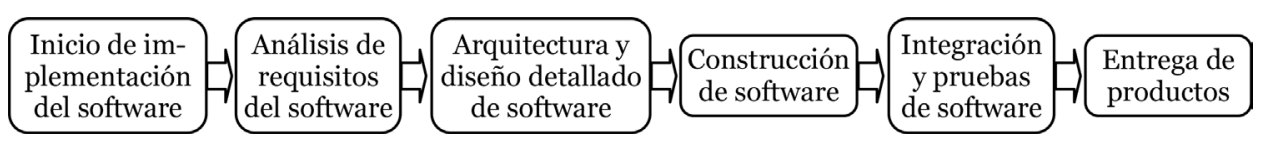

Figura 2 - Actividades principales del proceso de IS (ISO/IEC, 2011c)

\subsection{Modelos de factores en la mejora de procesos de software}

Las empresas que adoptan una mejora de procesos de software (MPS), se dice que puede considerarse como una innovación en tecnologías de la información (Anacona, Bastidas, Pino, \& Pardo, 2015). 
En este contexto, existen trabajos de investigación para pequeñas organizaciones (PO) como (Mejia Jezreel et al., 2012) y (M Jezreel et al., 2013), donde se proponen métodos para identificar los factores clave a considerar si la empresa desea implementar una mejora de procesos de software exitosa. Algunos factores identificados fueron: mantenimiento del software, calidad del producto y gestión del cambio.

Del mismo modo, en la literatura se han recopilado un conjunto de factores que pueden influir positiva o negativamente al realizar una MPS. Estos estudios incluyen: (i) El análisis realizado en (Cuevas, Calvo-Manzano, \& García, 2014) que muestra los puntos clave que deben tenerse en cuenta en las diferentes fases del desarrollo del software y en las personas que implementan el MPS. (ii) Los 18 factores de éxito identificados para lograr una mejora de procesos de software, en pequeñas y medianas empresas paquistaníes, descritas en (Sulayman, Mendes, Urquhart, Riaz, \& Tempero, 2014), en el contexto de aplicaciones de desarrollo web. (iii) El estudio de (Lee, Shiue, \& Chen, 2016) que analiza la influencia de otros factores como la cultura organizacional, la distribución del conocimiento entre los empleados, entre otros. (iv) Los inconvenientes asociados con la adopción de una metodología ágil descrita en (Paulk, 2011), donde se identifican un conjunto de factores tales como: el tamaño del proyecto, problemas culturales, entre otros. (v) El modelo conceptual de (Hameed et al., 2012), donde se recogieron 107 factores de la literatura actual, que pueden influir en la adopción de una innovación en tecnología de la información. Los factores se agruparon en 5 categorías: innovación (20 factores), organización (41 factores), entorno (16 factores), CEO (8 factores) y aceptación del usuario (22 factores).

\subsection{Trabajos relacionados}

Una de las ventajas de utilizar el estándar ISO/IEC 29110 es que permite a las empresas mejorar sus procesos de software sin tener la complejidad de otros estándares o modelos (Larrucea et al., 2010); en esta línea, hay estudios que analizan los potenciales beneficios y barreras de las pequeñas empresas en adoptar el estándar en un futuro, tales como (Basri \& O’Connor, 2010a) y (Sánchez-Gordón \& O’Connor, 2016). Además, en los artículos (O’Connor \& Laporte, 2011), (O’Connor \& Sanders, 2013), (Paucar, Laporte, Arteaga, \& Bruggmann, 2015) y (O’Connor, 2014) se estudian algunas buenas prácticas y directrices a tomar en cuenta cuando una pequeña organización adopta el estándar ISO/IEC 29110, tales como: "Paquetes de implementación", asistencia cercana al equipo para la implementación del estándar y el uso de herramientas de soporte para la gestión de procesos de IS y GP. Todos los artículos mencionados previamente, en esta sección, se consideran de gran relevancia pues contribuyen a comprender los factores que se analizan en el contexto de nuestro Proyecto ProCal-ProSer (Díaz et al., 2016).

Por otro lado, hay otro grupo de artículos que analizan los factores que influyen en las pequeñas empresas, al implementar una mejora de procesos de software utilizando el estándar ISO/IEC 29110. Uno de ellos (Wongsai, Siddoo, \& Wetprasit, 2015), recopila y clasifica 37 factores (de éxito y barreras de adopción) que pueden influenciar en la adopción del estándar ISO/IEC 29110. Nuestra investigación, a diferencia de la anterior, utiliza otro grupo de factores identificados en (Hameed et al., 2012) no necesariamente aplicados al estándar ISO/IEC 29110; sin embrago, en la sección de discusión, se analiza si se encontró alguna relación entre los factores identificados en (Wongsai et al., 2015) y los analizados en este estudio. 
Finalmente, los artículos de (Sanchez-Gordon, O’Connor, \& Colomo-Palacios, 2015) y (O’Connor, 2012) muestran el punto de vista de algunas PO con respecto a la adopción del estándar ISO/IEC 29110, la información obtenida a través del uso de la metodología de teoría fundamentada y entrevistas. Como resultado, se muestran factores que influyen en la adopción tales como: nivel de interés, nivel de aceptación y barreras presentes en una pequeña empresa para adoptar una mejora de proceso de software usando el estándar ISO/IEC 29110. Nuestra investigación tiene similitud con ambos estudios; sin embargo, el propósito de este estudio es conocer qué factores tienen una mayor o menor influencia en la mejora de los procesos de software (basado en el modelo conceptual de (Hameed et al., 2012)), a diferencia de los estudios previos que obtienen los factores como resultado de la metodología utilizada.

\section{Metodología de investigación}

En el presente estudio, se ha utilizado la metodología de investigación cualitativa "estudio de caso múltiple", debido a que se realiza un análisis exploratorio de la mejora de procesos en diferentes organizaciones, pero cada una como unidad de análisis independiente.

El protocolo que se ha seguido para el estudio de caso es el definido en (Genero, CruzLemus, \& Piattini, 2014), asimismo, se tiene como referencia el artículo de (Dávila \& Pessoa, 2015), que muestra un caso práctico de implementación del protocolo, en un análisis de factores de adopción en una compañía. El proceso seguido consta de 4 fases y varias etapas (ver Tabla 1).

\begin{tabular}{ll}
\hline Fase & Etapa \\
\hline & $(\mathrm{P} 1-1)$ Objetivo del estudio \\
& $(\mathrm{P} 1-2)$ Qué se estudia \\
P1: Diseño del estudio de caso & $\begin{array}{l}\text { (P1-4) Preoría requerida } \\
(\mathrm{P} 1-5) \text { Métodos de recolección } \\
(\mathrm{P} 1-6) \text { Selección de datos }\end{array}$ \\
& $(\mathrm{P} 2-1)$ Definición de cómo se realiza la recolección \\
\hline P2: Preparar recolección de la data & $(\mathrm{E} 1-1)$ Recolección de la data \\
\hline E1: Recolectar la data & $(\mathrm{E} 2-1)$ Análisis de la data \\
\hline E2: Analizar e interpretar la data & $(\mathrm{E} 2-2)$ Interpretación de la data \\
\hline R1: Reportar los resultados & $(\mathrm{R} 1-1)$ Llevar a cabo el informe \\
\hline
\end{tabular}

Tabla 1 - Fases y etapas del proceso del estudio de caso (Genero et al., 2014)

En los párrafos siguientes, se desarrollan las etapas del estudio de caso relacionado con la fase de planificación (P):

(P1-1) Objetivo del estudio: analizar cualitativamente la forma en que los factores del entorno influyen en la adopción del estándar ISO/IEC 29110. El trabajo se realiza en equipos de trabajo de cuatro PO, las cuales son descritas en la Sección 4. Para lograr el 
objetivo, se realizó un ciclo de mejora del proceso de software en todas las unidades de análisis utilizando el perfil básico del estándar ISO/IEC 29110, que involucra los procesos de implementación del software y gestión de proyectos. Dentro del proyecto ProCalProSer (Díaz et al., 2016), se estableció trabajar con modelos de factores utilizados en el contexto de mejora de procesos y modelos de factores de innovación tecnológica, con el objetivo de evaluar su aplicación en varias PO. Para este estudio en particular, se estableció trabajar con el modelo conceptual definido en (Hameed et al., 2012), que agrupa los factores de adopción en 5 categorías como se muestra en la sección 2.2. Este artículo usa y analiza los 16 factores del entorno definidos en el modelo y se propone como trabajo futuro el estudio del resto de los factores.

(P1-2) ¿Qué se estudia? las unidades de análisis son cuatro PO. Los equipos de trabajo tienen menos de 25 personas, por lo que no hubo restricciones para implementar la mejora del proceso basada en el estándar ISO/IEC 29110 en lo que respecta al perfil básico. La descripción detallada de las empresas y sus características se presenta en la Sección 4. En consecuencia, el objeto de estudio se basa en un grupo de instancias de análisis, cada una bajo su propio contexto, lo que permite definir un estudio de caso múltiple holístico (Genero et al., 2014).

(P1-3) Teoría requerida: ver la Sección 2.

(P1-4) Preguntas de investigación: la pregunta de investigación para el presente estudio es: ¿Cómo influyen los factores del entorno en la adopción de las prácticas de perfil básico definidas en la ISO/IEC 29110-5-1-2?

(P1-5) Métodos de recolección: la información recopilada fue de tipo cualitativo y se obtuvo en dos etapas. (i) Primera etapa: por cada empresa se realizó una evaluación diagnóstica, utilizando documentos e informes, para comparar el nivel de cumplimiento de sus prácticas versus las definidas en el estándar ISO/IEC 29110-5-1-2, tanto al inicio como al final de la mejora. El proceso de recopilación de datos, para esta primera etapa, se puede considerar de tercer grado, de acuerdo con lo especificado en (Genero et al., 2014). (ii) Segunda etapa: en todas las empresas se realizaron entrevistas semiestructuras, para lograr un estudio descriptivo y exploratorio de los factores del entorno que influyeron en la mejora de procesos de software. Las preguntas de la entrevista fueron formuladas en base a la descripción de cada factor y fueron revisadas y aprobadas por un revisor externo con más de 10 años de experiencia en cuestiones de MPS y calidad de los procesos de software. Detalles adicionales de la entrevista se muestran en la Sección 5 .

(P1-6) Selección de datos: se realizó en dos etapas acorde a (P1-5). (i) Primera etapa: el informe del nivel de adopción basado en la evaluación de la ISO/IEC 15504 y realizado por un evaluador externo, el cual pudo determinar qué prácticas del estándar fueron adoptados y cuáles no, y hasta qué punto (ver Sección 5). (ii) Segunda etapa: las entrevistas fueron refinadas y consolidadas para obtener un diagnóstico de los factores relacionados al entorno que influenciaron en la adopción de la norma (ver la Sección 5).

(P2-1) Definición de cómo se realiza la recolección: se llevó a cabo en dos etapas. La primera, donde se evaluó el nivel de adopción del estándar y la segunda, a través de entrevistas semiestructuradas, cuyo foco central fue conocer qué factores del modelo de (Hameed et al., 2012) influyeron positiva o negativamente en la adopción del perfil básico. 
Los pasos restantes se analizaron de la siguiente manera: (E1-1), (E2-1) y (E2-2) en la sección 5, (R1-1) en todo el artículo.

\section{Unidades de análisis}

Se tienen cuatro unidades de análisis para el estudio de caso múltiple. Por cuestiones de confidencialidad, las empresas se llamaron alfa, beta, gamma y delta, las cuales se describen en la Tabla 2.

\begin{tabular}{|c|c|c|c|c|}
\hline Ítem & Alpha & Beta & Gamma & Delta \\
\hline Descripción & $\begin{array}{l}\text { Área de software } \\
\text { dentro de una } \\
\text { organización } \\
\text { educativa. Desarrolla } \\
\text { proyectos a ser } \\
\text { utilizados en } \\
\text { entornos educativos. }\end{array}$ & $\begin{array}{l}\text { PO del sector } \\
\text { privado que } \\
\text { desarrolla } \\
\text { soluciones de } \\
\text { hardware, software } \\
\text { y redes para la } \\
\text { industria del juego. }\end{array}$ & $\begin{array}{l}\text { PO que ofrece } \\
\text { soluciones en } \\
\text { diversos sectores, } \\
\text { principalmente } \\
\text { comerciales, } \\
\text { minoristas, de salud, } \\
\text { minería, ingeniería y } \\
\text { educación. }\end{array}$ & $\begin{array}{l}\text { PO que ofrece } \\
\text { soluciones } \\
\text { para iPhone, } \\
\text { iPad, Android y } \\
\text { Windows Phone. }\end{array}$ \\
\hline Equipo & $\begin{array}{l}1 \text { gerente de } \\
\text { proyecto, } 2 \\
\text { desarrolladores } \\
\text { junior y } 1 \text { diseñador } \\
\text { gráfico }\end{array}$ & $\begin{array}{l}1 \text { gerente de } \\
\text { proyecto, } 3 \\
\text { desarrolladores } \\
\text { senior y } 1 \text { junio, } 2 \\
\text { analistas y } 1 \text { gerente } \\
\text { de pruebas }\end{array}$ & $\begin{array}{l}1 \text { gerente de proyecto, } \\
2 \text { analistas y } 3 \\
\text { desarrolladores senior }\end{array}$ & $\begin{array}{l}1 \text { gerente general, } \\
1 \text { subgerente, } 4 \\
\text { desarrolladores } \\
\text { senior y } 2 \\
\text { administrativos. }\end{array}$ \\
\hline $\begin{array}{l}\text { Experiencia } \\
\text { empresarial }\end{array}$ & 5 años & 13 años & 8 años & 10 años \\
\hline $\begin{array}{l}\text { Experiencia } \\
\text { en MPS }\end{array}$ & $\begin{array}{l}\text { Sin experiencia } \\
\text { previa }\end{array}$ & $\begin{array}{l}\text { Sin experiencia } \\
\text { previa }\end{array}$ & Sin experiencia previa & $\begin{array}{l}\text { Sin experiencia } \\
\text { previa }\end{array}$ \\
\hline $\begin{array}{l}\text { Apoyo } \\
\text { externo en } \\
\text { MPS }\end{array}$ & $\begin{array}{l}\text { Estudiante de } \\
\text { posgrado }\end{array}$ & $\begin{array}{l}\text { Estudiante de } \\
\text { posgrado }\end{array}$ & $\begin{array}{l}\text { Estudiante } \\
\text { universitario } \\
\text { (remunerado) }\end{array}$ & $\begin{array}{l}\text { Estudiante } \\
\text { universitario } \\
\text { (remunerado) }\end{array}$ \\
\hline $\begin{array}{l}\text { Duración de } \\
\text { la MPS }\end{array}$ & 4 meses & 6 meses & 6 meses & 6 meses \\
\hline
\end{tabular}

Tabla 2 - Unidades de análisis

\section{Análisis y resultados}

En esta sección se analizan los resultados obtenidos del nivel de adopción a la norma y los factores que influenciaron.

\subsection{Análisis del nivel de adopción}

El proceso de recolección de datos empezó con una evaluación diagnóstica al inicio del proyecto, en la cual se evaluaron los procesos GP y IS (del perfil básico) para conocer el estado actual de las empresas y establecer sus propuestas de mejora. La evaluación se realizó utilizando la ISO/IEC 15504-2, como se especifica en (ISO/IEC, 2011b). 
La evaluación diagnóstica fue realizada por un evaluador externo calificado junto con el gerente de proyecto de cada compañía y presentando la documentación requerida por cada práctica o grupo de prácticas.

Como resultado de la evaluación inicial se obtuvo el grado de adherencia de las empresas en cada uno de los procesos implementados, en base a cuatro niveles definidos por la ISO/IEC 15504-2: N (no cumplido), P (parcialmente cumplido), L (ampliamente cumplido) y F (totalmente logrado). Asimismo, se colocaron porcentajes de adhesión a la guía; aquellos que representan el grado de cumplimiento basado en las prácticas adoptadas por la empresa. Estos porcentajes fueron colocados por los autores para facilitar la comparación y el análisis entre la evaluación inicial y la final. La calificación práctica básica del proceso de evaluación del proceso se muestra en el Apéndice A.

Las metas de mejora se establecieron en base a la evaluación diagnóstica y fueron diferentes para cada empresa: Alfa tuvo como objetivo alcanzar al menos un nivel L en ambos procesos del perfil básico; mientras que Beta pretendía alcanzar un nivel P en GP y un nivel L en IS y finalmente, Gamma y Delta apuntaban a alcanzar el nivel F en IS y GP. La implementación se llevó a cabo en todas las empresas utilizando el perfil básico (GP y IS) de la ISO/IEC 29110-5-1-2.

El proceso seguido para la evaluación inicial fue replicado para la evaluación final y los resultados se presentan en las columnas EI y EF en la Tabla 3. Se puede observar en dicha Tabla, que Alfa logró una mejora significativa en GP, pasando de una calificación $\mathrm{N}$ con un cumplimiento del $10.6 \%$ a una L con $62.5 \%$.

\begin{tabular}{|c|c|c|c|c|c|c|}
\hline \multirow[b]{2}{*}{ Id Org. } & \multicolumn{3}{|c|}{ Gestión de proyectos } & \multicolumn{3}{|c|}{ Implementación del software } \\
\hline & $\begin{array}{l}\text { Adherencia } \\
\text { (\%) }\end{array}$ & $\begin{array}{l}\text { Variación } \\
\text { de nivel }\end{array}$ & $\begin{array}{l}\text { Variación } \\
\text { de } \\
\text { capacidad }\end{array}$ & $\begin{array}{l}\text { Adherencia } \\
\text { (\%) }\end{array}$ & $\begin{array}{l}\text { Variación } \\
\text { de nivel }\end{array}$ & $\begin{array}{l}\text { Variación } \\
\text { de } \\
\text { capacidad }\end{array}$ \\
\hline & $\mathbf{E I} \rightarrow \mathbf{E F}$ & $\mathbf{E I} \rightarrow \mathbf{E F}$ & $\mathbf{E I} \rightarrow \mathbf{E F}$ & $\mathbf{E I} \rightarrow \mathbf{E F}$ & $\mathbf{E I} \rightarrow \mathbf{E F}$ & $\mathbf{E I} \rightarrow \mathbf{E F}$ \\
\hline Alfa & $10.6 \rightarrow 62.5$ & $\mathrm{~N} \rightarrow \mathrm{L}$ & $\mathrm{o} \rightarrow \mathrm{o}$ & $37.9 \rightarrow 42.8$ & $\mathrm{P} \rightarrow \mathrm{L}$ & $0 \rightarrow 0$ \\
\hline Beta & $14.6 \rightarrow 48.1$ & $\mathrm{~N} \rightarrow \mathrm{P}$ & $\mathrm{o} \rightarrow \mathrm{o}$ & $21.9 \rightarrow 49.4$ & $\mathrm{P} \rightarrow \mathrm{P}$ & $0 \rightarrow 0$ \\
\hline Gamma & $89.2 \rightarrow 96.2$ & $\mathrm{~F} \rightarrow \mathrm{F}$ & $1 \rightarrow 1$ & $52.9 \rightarrow 71.8$ & $\mathrm{~L} \rightarrow \mathrm{L}$ & $0 \rightarrow 0$ \\
\hline Delta & $27.9 \rightarrow 84.6$ & $\mathrm{P} \rightarrow \mathrm{L}$ & $0 \rightarrow 1$ & $29.9 \rightarrow 77.4$ & $\mathrm{P} \rightarrow \mathrm{L}$ & $0 \rightarrow 0$ \\
\hline
\end{tabular}

Tabla 3 - Comparación de la evaluación inicial y final

\subsection{Análisis de los factores}

Después de la evaluación inicial y final, se analizaron los factores que influenciaron en la adopción a través de una entrevista semiestructurada (ver Sección 3, P1-5), la cual se realizó a los gerentes de proyecto de cada empresa. Los factores relacionados con el entorno se muestran en la Tabla 4, junto con una breve descripción. Para cada factor, 
el investigador propuso una pregunta que permitiría saber durante la entrevista si el factor influenció en la mejora de procesos de software y, de ser así, en qué nivel. Estas preguntas se muestran en el Apéndice B.

\begin{tabular}{|c|c|c|}
\hline ID & Factor & Descripción \\
\hline EO1 & Presión competitiva & Presión ejercida por los competidores del mismo rubro. \\
\hline EO2 & Presión externa & $\begin{array}{l}\text { Influencias que surgen de varias fuentes dentro del entorno compet- } \\
\text { itivo que rodea a la organización. }\end{array}$ \\
\hline EO3 & Apoyo del gobierno & $\begin{array}{l}\text { El gobierno apoya con incentivos financieros, reducción de impues- } \\
\text { tos, proyectos pilotos para beneficiar la innovación. }\end{array}$ \\
\hline EO4 & Soporte del vendedor & $\begin{array}{l}\text { Asistencia del vendedor de un producto o servicio con el que cuenta } \\
\text { la empresa. }\end{array}$ \\
\hline EO5 & Soporte de los socios & $\begin{array}{l}\text { Grado de Apoyo de los socios de la empresa a las decisiones corpo- } \\
\text { rativas. }\end{array}$ \\
\hline Eo6 & Disposición de los socios & Disponibilidad de los socios de apoyar la mejora de procesos. \\
\hline EO7 & Incertidumbre del entorno & $\begin{array}{l}\text { La empresa tiene poca información sobre su entorno externo, por lo } \\
\text { tanto, su comportamiento es errático. }\end{array}$ \\
\hline Eo8 & Vinculación vertical & $\begin{array}{l}\text { Actividades de coordinación y comunicación desde los puestos más } \\
\text { altos hasta los más bajos de la empresa. }\end{array}$ \\
\hline Eo9 & Defensa de los socios & Los socios de la empresa apoyan la mejora de procesos. \\
\hline E1O & Presión del gobierno & $\begin{array}{l}\text { Presión ejercida por el gobierno hacia la adopción de estándares y la } \\
\text { obtención de certificaciones. }\end{array}$ \\
\hline E11 & Número de competidores & Cantidad de competidores identificados por la empresa. \\
\hline E12 & Experiencia externa & La empresa solicita consultoría externa de un proveedor externo. \\
\hline E13 & Eficacia de la consultoría & $\begin{array}{l}\text { La consultoría solicitada brindó una solución y/o ayudó a esclarecer } \\
\text { los problemas presentes. }\end{array}$ \\
\hline E14 & Confianza en los socios & $\begin{array}{l}\text { Confianza brindada por los socios en el proceso de adopción de la } \\
\text { mejora de procesos. }\end{array}$ \\
\hline E15 & Globalización & $\begin{array}{l}\text { Mayor cantidad de empresas de software en el mundo, clientes de la } \\
\text { empresa en otros países, nuevos mercados. }\end{array}$ \\
\hline E16 & Influencia social & $\begin{array}{l}\text { La percepción del público (u otra empresa) sobre la posición de la } \\
\text { empresa. }\end{array}$ \\
\hline
\end{tabular}

Tabla 4 - Factores del entorno

Asimismo, cada factor fue evaluado de acuerdo con los siguientes criterios: (i) Influencia en la mejora del proceso: positiva o negativa. (ii) Nivel de influencia, en una escala de Likert, de 1 a 5 , donde 1 es influencia muy baja y 5 una influencia muy alta. (iii) Justificación de la influencia del factor. (iv) ¿qué problemas se presentaron sobre este factor durante la mejora? (v) Criticidad del problema, en una escala Likert, de 1 a 5 , donde 1 es muy baja criticidad y 5 muy alta criticidad. (vi) ¿cómo lidió con este problema?

La Tabla 5 presenta el consolidado de las entrevistas, considerando dos columnas para cada empresa: el tipo de influencia (Inf.) y el nivel de esa influencia (Nivel). Si el factor 
evaluado no tuvo influencia, se coloca N.A, si la influencia fue positiva se coloca (+) y si fue negativa se coloca (-). En esta investigación utilizamos la síntesis narrativa, de acuerdo con lo establecido en (Popay et al., 2006), para analizar la información recolectada de los factores del entorno, que a través de entrevistas, fueron recopiladas y mapeadas en la Tabla 5 .

\begin{tabular}{lcccccccc}
\hline & \multicolumn{2}{c}{ Alfa } & \multicolumn{2}{c}{ Beta } & \multicolumn{2}{c}{ Gamma } & \multicolumn{2}{c}{ Delta } \\
Factor ID & Inf. & Nivel & Inf. & Nivel & Inf. & Nivel & Inf. & Nivel \\
\hline$E O 1$ & N.A. & N.A. & N.A. & N.A. & N.A. & N.A. & N.A. & N.A. \\
\hline$E O 2$ & + & 4 & + & 3 & N.A. & N.A. & N.A. & N.A. \\
\hline$E O 3$ & + & 3 & N.A. & N.A. & N.A. & N.A. & N.A. & N.A. \\
\hline$E O 4$ & + & 1 & N.A. & N.A. & N.A. & N.A. & N.A. & N.A. \\
\hline$E O 5$ & + & 3 & + & 5 & + & 2 & + & 5 \\
\hline$E O 6$ & + & 3 & N.A. & N.A. & + & 3 & + & 5 \\
\hline$E O 7$ & N.A. & N.A. & + & 1 & N.A. & N.A. & N.A. & N.A. \\
\hline$E O 8$ & + & 4 & + & 4 & + & 5 & + & 5 \\
\hline$E O 9$ & - & 2 & - & 3 & + & 4 & + & 3 \\
\hline$E 10$ & N.A. & N.A. & + & 2 & + & 2 & N.A. & N.A. \\
\hline$E 11$ & N.A. & N.A. & + & 2 & N.A. & N.A. & N.A. & N.A. \\
\hline$E 12$ & N.A. & N.A. & N.A. & N.A. & N.A. & N.A. & N.A. & N.A. \\
\hline$E 13$ & N.A. & N.A. & N.A. & N.A. & N.A. & N.A. & N.A. & N.A. \\
\hline$E 14$ & + & 5 & + & 4 & + & 3 & + & 4 \\
\hline$E 15$ & + & 1 & + & 2 & + & 3 & + & 4 \\
\hline$E 16$ & N.A. & N.A. & N.A. & N.A. & + & 5 & N.A. & N.A. \\
\hline & & & & & & & &
\end{tabular}

Tabla 5 - Resultado de la evaluación de factores

En base a los resultados obtenidos, se analizaron los factores, de manera individual o agrupados por similitud de respuestas:

1. Los factores E01, E12 y E13, no influenciaron en ninguna empresa.

2. El factor E02, presión externa, no tuvo influencia en Gamma ni Delta, pero sí en Alfa y Beta. No se puede generalizar una razón para explicar este resultado por ambas empresas, pero sí, por separado. Alfa suele postular sus proyectos como parte de convocatorias al Estado, lo cual se ve reflejado en Eo3, por lo cual existe influencia competitiva del entorno en su MPS. Para Beta, la influencia viene de sus competidores, tal como se ve en relación con el factor E11, siendo esta la única PO que tuvo esa influencia.

3. El factor EO3 y Eo4 solo influenciaron en Alfa, lo cual está acorde a su propio contexto pues es una unidad de software dentro de una entidad educativa; mientras que las otras PO, son desarrolladoras de software.

4. Los factores E05, E08, E09, E14 y E15, han influenciado en todas las empresas sin importar el contexto donde se desempañaba. En particular, en Alfa el factor 
E14 tuvo un impacto positivo muy alto y el comentario al respecto fue: "la razón es que el líder técnico es también el especialista en software y el gerente de área confió plenamente sobre sus capacidades para implementar la mejora en los procesos y obtener los resultados buscados".

5. El único factor que influenció negativamente en el estudio fue Eog y esto sucedió en las empresas Alfa y Beta. Al analizar un extracto de la entrevista a la compañía Beta con respecto a este factor se tuvo: "El gerente de la empresa sabía sobre la mejora de los procesos, pero al no ser una persona técnica (no era ingeniero), prefirió no entrar en los detalles de la implementación de la mejora y lo dejó en manos del líder del proyecto". Por este motivo, al no apoyar ni estar involucrado el gerente directamente la MPS, generó retrasos en la implementación de la mejora.

\section{5•3. Amenazas del estudio}

Se debe considerar que la mejora de procesos fue guiada por un miembro del equipo del proyecto ProCal-ProSer en gamma y delta, lo que no sucedió en alfa y beta. Del mismo modo, con respecto al análisis de los factores, las entrevistas se hicieron a los líderes del equipo, tal vez los miembros del equipo tenían una opinión diferente y se hubiera obtenido otros niveles de influencia para los mismos factores.

\section{Discusión final y trabajo futuro}

La adopción de una mejora de procesos de software implica una serie de pasos y buenas prácticas que deben seguirse para alcanzar los objetivos establecidos. En el caso de pequeñas empresas, en base a los estudios previos y los resultados obtenidos, la mejora de procesos tendrá mayor éxito si existe un compromiso total desde los cargos más altos de la empresa hasta los más bajos.

Luego de analizar los 16 factores del entorno, se puede concluir que, 5 de ellos influyeron en todas las organizaciones, independientemente del contexto en el que se encontraban. Dentro de estos cinco factores, tres de ellos, E05 soporte de los socios, Eo8 vinculación vertical y E14 confianza en los socios, están relacionados fuertemente con el apoyo de la alta gerencia a la mejora de procesos de software, lo cual está acorde con la investigación de (Wongsai et al., 2015) (Sección 2.2), donde dos factores mapeados, "compromiso y participación” y “política organizacional”, ambos relacionados también con el apoyo de la alta gerencia, se consideran factores de éxito. Además, estos tres factores también están relacionados con la investigación de (Sanchez-Gordon et al., 2015) donde se menciona que la "falta de necesidad percibida" y "necesidad de recursos" son barreras para lograr la adopción de una mejora de procesos. En este estudio, todas las empresas pudieron superar estas barreras pues contaban con los recursos para implementar la mejora de procesos.

Los otros dos factores que influenciaron en todas las empresas, Eog defensa de los socios y E15 globalización, no han sido identificados previamente como factores de éxito o barreras de adopción en ningún artículo revisado, por lo tanto, ambos deberían tomarse en consideración al realizar una MPS o estudios similares. De los dos antes mencionados, tomar particular atención a Eog defensa de los socios, pues fue el único que influenció negativamente en dos empresas. De igual forma, se ha podido verificar que algunos factores están relacionados con otros factores por el contexto particular de 
la empresa, como es el caso de E02, que tuvo influencia positiva en dos empresas y la razón era su relación con Eo3 en Alfa y con E11 en Beta.

Como trabajo futuro, se propone realizar el procedimiento descrito en este documento, pero centrándose en los otros 4 grupos de factores del modelo de (Hameed et al., 2012): innovación, organización, CEO y aceptación del usuario. Además, se propone realizar un análisis de correlación entre el grado de adopción después de la mejora de procesos de software y los factores que han influenciado en la adopción de la mejora; así como, una posible correlación entre los mismos factores.

\section{Agradecimientos}

Este trabajo ha sido realizado dentro del proyecto ProCal-ProSer financiado por Innóvate Perú bajo el Contrato 210 - FINCYT-IA-2013 y por el Departamento de Ingeniería y el Grupo de Investigación y Desarrollo de Ingeniería de Software (GIDIS) de la Pontificia Universidad Católica del Perú.

\section{Referencias}

Anacona, D., Bastidas, M. I., Pino, F. J., \& Pardo, C. (2015). Innova-Procedure : A procedure to guide the innovation of software development processes in VSEs. In Proceedings of Computing Colombian Conference (1oCCC) $201510^{\text {th }}$, (pp. 108-115). Bogota, Colombia: IEEE.

Basri, S., \& O’Connor, R. V. (2010a). Understanding the perception of very small software companies towards the adoption of process standards. In Communications in Computer and Information Science book (vol. 99, (pp. 153-164). Berlin: Springer. https://doi.org/10.1007/978-3-642-15666-3_14

Basri, S. Bin, \& O’Connor, R. V. (2010b). Organizational commitment towards software process improvement: An Irish software VSEs case study. In Proceedings of International Symposium on Information Technology - System Development and Application and Knowledge Society, ITSim'1o (Vol. 3, pp. 1456-1461). Kuala Lumpur, Malaysia: IEEE. https://doi.org/10.1109/ITSIM.2010.5561489

Bayona, S., Calvo-Manzano, J. A., \& Feliu, T. S. (2013). Review of Critical Success Factors Related to People in Software Process Improvement. In Proceedings of 2oth EuroSPI Conference (pp. 179-189). Berlin: Springer. https://doi.org/10.1007/9783-642-39179-8_16

Bayona, S., Calvo-Manzano, J., Cuevas, G., \& Feliu, T. S. (2010). Taxonomia de Factores Criticos para el Despliegue de Procesos Software. REICIS, Revista Española de Innovación, Calidad E Ingeniería Del Software, 6(3), 6-22. Retrieved from http://oa.upm.es/8242/

Brodman, J., \& Johnson, D. (1997). A Software Process Improvement Approach Tailored for Small Organizations and Small Projects. In Proceedings of 19th International Conference on Software Engineering (pp. 661-662). Boston, MA, USA: ICSE. https://doi.org/10.1109/ICSE.1997.610455 
Cano, C., Melgar, A., Dávila, A., \& Pessoa, M. (2015). Comparison of Software Process Models: A Systematic Literature Review. In Proceedings of XLI Conferencia Latinoamericana en Informática (CLEI 2015) (pp. 19-23). Arequipa, Peru: SPC.

Cuevas, G., Calvo-Manzano, J. A., \& García, I. (2014). Some Key Topics to be Considered in Software Process Improvement (pp. 119-142). https://doi.org/10.4018/978-14666-5182-1.choo8

Dávila, A., \& Pessoa, M. (2015). Factors driving the adoption of ISO/IEC 29110: A case study of a small software enterprise. In Proceedings of 2015 41st Latin American Computing Conference, CLEI 2015 (pp. 1-8). Piscataway, New Jersey, USA: IEEE. https://doi.org/10.1109/CLEI.2015.7360042

Díaz, A., De Jesús, C., Melendez, K., \& Dávila, A. (2016). ISO/IEC 29110 Implementation on two Very Small Software Development Companies in Lima: Lessons Learned. IEEE Latin America Transactions, 14(5), 2504-2510. Retrieved from https://sites. google.com/a/pucp.pe/procal-proser/

Ebert, C. (1999). Technical controlling and software process improvement. Journal of Systems and Software, 46, 25-39. https://doi.org/http://dx.doi.org/10.1016/ So164-1212(98)10086-9

Elhag, A., Elshaikh, M. A., Mohamed, R., \& Babar, M. I. (2013). Problems and future trends of software process improvement in some Sudanese software organizations. In Proceedings of International Conference on Computer, Electrical and Electronics Engineering. ICCEEE 2013 (pp. 263-268). Khartoum, Sudan: IEEE. https://doi.org/10.1109/ICCEEE.2013.6633945

Genero, M., Cruz-Lemus, J., \& Piattini, M. (2014). Métodos de investigación en ingeniería del software. Madrid: RA-MA Editorial.

Hameed, M. A., Counsell, S., \& Swift, S. (2012). A conceptual model for the process of IT innovation adoption in organizations. Journal of Engineering and Technology Management - JET-M, 29(3), 358-390. https://doi.org/10.1016/j. jengtecman.2012.03.007

Horvat, R. V., Rozman, I., \& Györkös, J. (2000). Managing the complexity of SPI in small companies. Software Process: Improvement and Practice, 5, 45-54. https://doi.org/10.1002/(SICI)1099-1670(200003)5:1<45::AID-SPIP110>3.0.CO;2-2

Huang,D.,\&Zhang,W.(2010).CMMIinMedium\&SmallEnterprises:ProblemsandSolutions. In Proceedings of 2nd IEEE International Conference on Information Management and Engineering (pp. 171-174). https://doi.org/10.1109/ICIME.2010.5478220

Humphrey, W. S. (1995). A Discipline for Software Engineering. Boston, MA, USA: Addison-Wesley Longman Publishing Co., Inc. Retrieved from https://books. google.com.pe/books?id=MvnSAwAAQBAJ\&lpg=PA3\&dq=discipline of software The State of the House\&pg $=\mathrm{PA} 3 \# \mathrm{v}=$ onepage $\& \mathrm{q} \& \mathrm{f}=$ false

ISO/IEC. (2008). ISO/IEC 12207: 2008 System and Software Engineering - Software Life Cycle Processes. Geneva: ISO/IEC. Retrieved from https://www.iso.org/obp/ ui/\#iso:std:iso-iec:12207:ed-2:v1:en 
ISO/IEC. (2011a). ISO/IEC TR 2911O-1 Lifecycle profiles for Very Small Entities (VSEs ) - Part 1: Overview. Geneva: ISO/IEC.

ISO/IEC. (2011b). ISO/IEC TR 2911O-1 Lifecycle profiles for Very Small Entities (VSEs ) - Part 3: Assessment guide. Geneva: ISO/IEC.

ISO/IEC. (2011c). ISO/IEC TR 2911O-1 Lifecycle profiles for Very Small Entities (VSEs ) - Part 5-1-2:Management and engineering guide: Generic profile group: Basic profile (Vol. 2011). Geneva: ISO/IEC.

Jezreel, M., Mirna, M., Edrisi, M., Diego, E., Al Rumeni, A., \& Juan, M. (2013). Leading the effort of software process improvement in SMEs. In Proceedings of 8th Iberian Conference on Information Systems and Technologies (CISTI) (pp. 1-7). Lisbon, Portugal: IEEE.

Jezreel, M., Mirna, M., Pablo, N., Edgar, O., Alejandro, G., \& Sandra, M. (2012). Identifying findings for software process improvement in SMEs: An experience. In Porceedings of 9th Electronics, Robotics and Automotive Mechanics Conference, CERMA 2012 (pp. 141-146). Cuernavaca, Mexico: Curran Associates, Inc. (https://doi.org/10.1109/CERMA.2012.30

Kalinowski, M., Weber, K., Santos, G., Franco, N., Duarte, V., \& Travassos, G. (2015). Software Process Improvement Results in Brazil Based on the MPS-SW Model. Software Quality Professional, 17(4), 15-28.

Laporte, C. Y., O’Connor, R. V., \& Paucar, L. H. G. (2016). The implementation of ISO/ IEC 29110 software engineering standards and guides in very small entities. In Evaluation of Novel Approaches to Software Engineering (Vol. 599, pp. 162-179). Berlin: Springer. https://doi.org/10.1007/978-3-319-30243-O_9

Larrucea, X., O’Connor, R. V., Colomo-Palacios, R., \& Laporte, C. Y. (2010). Software Process Improvement in Very Small Organizations. IEEE Software, 33(April), 85-89. https://doi.org/10.1109/MS.2016.42

Lee, J.-C., Shiue, Y.-C., \& Chen, C.-Y. (2016). Examining the impacts of organizational culture and top management support of knowledge sharing on the success of software process improvement. Computers in Human Behavior, 54, 462-474. https://doi.org/10.1016/j.chb.2015.08.030

Lucho, S., Melendez, K., \& Dávila, A. (2018). Analysis of environmental factors in the adoption of ISO/IEC 29110. Multiple case study. In: J. Mejia, M. Muñoz, Á. Rocha, Y. Quiñonez, \& J. Calvo-Manzano (Eds.), Trends and Applications in Software Engineering (pp. 82-93). Cham: Springer International Publishing.

Miramontes, J., Muñoz, M., Calvo-Manzano, J. A., \& Corona, B. (2016). Establecimiento del estado del arte sobre el aligeramiento de procesos de software. RISTI - Revista Ibérica de Sistemas E Tecnologias de Informação, (17), 16-25. https://doi.org/10.17013/risti.17.16-25

Nirmala, D., \& LathaMaheswari, T. (2016). Automated testcase generation for software quality assurance. In Porceedings of 1oth International Conference on Intelligent Systems and Control (ISCO) (pp. 1-6). India: IEEE. https://doi.org/10.1109/ISCO.2016.7727003 
O’Connor, R. V. (2012). Evaluating management sentiment towards ISO/IEC 29110 in very small software development companies. Communications in Computer and Information Science, 290, 277-281. https://doi.org/10.1007/978-3-642-30439-2_31

O’Connor, R. V. (2014). Early Stage Adoption of ISO/IEC 29110 Software Project Management Practices: A Case Study. In Proceedings of International Conference on Software Process Improvement and Capability Determination (pp. 226-237). Dublin, Ireland: Springer. https://doi.org/10.1007/978-3-319-13036-1_20

O'Connor, R. V., \& Laporte, C. Y. (2011). Using ISO/IEC 29110 to harness process improvement in very small entities. In Proceedings of 18th European Software Process Improvement Conference. (Vol. 172, pp. 225-235). Roskilde, Denmark: Springer. https://doi.org/10.1007/978-3-642-22206-1

O'Connor, R. V., \& Sanders, M. (2013). Lessons from a Pilot Implementation of ISO/IEC 29110 in a Group of Very Small Irish Companies. In Proceedings of 13th International Conference on Software Process Improvement and Capability dEtermination (SPICE) (pp. 243-246). Bremen, Germany: Springer. https://doi.org/10.1007/978-3-642-38833-0_23

Oktaba, H., Alquicira, C., Su, A., Martinez, A., Quintanilla, G., Mara, R., \& Flores, M. (2005). Modelo de Procesos para la Industria de Software: MoProSoft. Mexico: Secretaría de Economía.

Paucar, L. G., Laporte, C. Y., Arteaga, J., \& Bruggmann, M. (2015). Implementation and Certification of ISO/IEC 29110 in an IT Startup in Peru. Software Quality Professional, 17(2), 16-29.

Paulk, M. C. (2011). On Empirical Research into Scrum Adoption. Pittsburgh: Carnegie Mellon University.

Piattini, M. (2008). Competisoft. Mejora de procesos software para pequeñas y medianas empresas y proyectos. Madrid: RA-MA Editorial.

Popay, J., Roberts, H., Sowden, A., Petticrew, M., Arai, L., Rodgers, M., \&Duffy, S. (2006). Guidance on the Conduct of Narrative Synthesis in Systematic Reviews. A Product from the ESRC Methods Programme. Lancaster: Lancaster University https://doi.org/10.1111/j.1523-536x.1995tboo261.x

Razón, O. C., Durán, J. G., González, J. A. G., Cortérights, F. I., \& Miranda, J. M. (2014). Cómo Guiar a las Pymes en la Mejora de Procesos Software. RISTI - Revista Ibérica de Sistemas E Tecnologias de Informação, (spe1), 17-30. https://doi.org/10.4304/risti.e1.17-30

Ruiz, J. C., Osorio, Z., Mejia, J., Muñoz, M., Chávez, A. M., \& Olivares, B. (2011). Definition of a hybrid measurement process for the models ISO/IEC 15504-ISO/IEC 12207:2008 and CMMI Dev 1.3 in SMEs. In Proceedings of Electronics, Robotics and Automotive Mechanics Conference (pp. 421-426). Cuernavaca, Mexico: IEEE. https://doi.org/10.1109/CERMA.2011.74

Sánchez-Gordón, M. L., \& O’Connor, R. V. (2016). Understanding the gap between software process practices and actual practice in very small companies. Software Quality Journal, 24(3), 549-570. https://doi.org/10.1007/s11219-015-9282-6 
Sanchez-Gordon, M. L., O’Connor, R. V., \& Colomo-Palacios, R. (2015). Evaluating VSES viewpoint and sentiment towards the ISO/IEC 29110 standard: A two Country grounded theory study. Communications in Computer and Information Science, 526, 114-127. https://doi.org/10.1007/978-3-319-19860-6_10

SEI (2010). CMMI ® para Desarrollo, Versión 1.3. Software Engineering Institute. Retrieved from http://www.sei.cmu.edu/library/assets/whitepapers/Spanish Technical Report CMMI V 1 3.pdf

Sulayman, M., Mendes, E., Urquhart, C., Riaz, M., \& Tempero, E. (2014). Towards a theoretical framework of SPI success factors for small and medium web companies. Information and Software Technology, 56(7), 807-820. https://doi.org/10.1016/j. infsof.2014.02.006

Sulayman, M., Urquhart, C., Mendes, E., \& Seidel, S. (2012). Software process improvement success factors for small and medium Web companies: A qualitative study. Information and Software Technology, 54(5), 479-500. https://doi.org/10.1016/j.infsof.2011.12.007

Svensson, R. B., Gorschek, T., Regnell, B., Torkar, R., Shahrokni, A., \& Feldt, R. (2012). Quality Requirements in Industrial Practice - An Extended Interview Study at Eleven Companies. IEEE Transactions on Software Engineering, 38(4), 923-935. https://doi.org/10.1109/tse.2011.47

Unterkalmsteiner, M., Gorschek, T., Islam, M., Cheng, C. K., Permadi, R., \& Feldt, R. (2012). Evaluation and Measurement of Software Process Improvement-A Systematic Literature Review. IEEE Transactions on Software Engineering, 38(2), 398-424. https://doi.org/10.1109/TSE.2011.26

Vasconcellos, F., Landre, G., Cunha, J., Oliveira, J. L., Ferreira, R. A., \& Vincenzi, A. M. (2016). Approaches to strategic alignment of software process improvement: A systematic literature review. Journal of Systems and Software, 123, 45-63. https://doi.org/10.1016/j.jss.2016.09.030

Wongsai, N., Siddoo, V., \& Wetprasit, R. (2015). Factors of influence in software process improvement: An ISO/IEC 29110 for very-small entities. In 7 th International Conference on Information Technology and Electrical Engineering: Envisioning the Trend of Computer, Information and Engineering, ICITEE (pp. 12-17).Chiang Mai, Thailand: IEEE.

\section{Apéndice.}

Disponible en:

https://drive.google.com/drive/folders/oB_K_llz5juqPMll5TC1sNkliWGc 